DOI: http://dx.doi.org/10.18569/tempus.v14i1.2624

\title{
Baixa cobertura de saúde bucal: como potencializar o cuidado?
}

\section{Low Oral Health Coverage: How to Improve Care?}

\section{Un Informe de Experiencia del Proyecto de Extensión de Cuidado Dental Vinculado al Sistema Único de Salud de Brasil}

Maria Laura Braccini Fagundes ${ }^{1}$ Orlando Luiz do Amaral Júnior Ariane Soares Krassmann Patrícia Bastianello Campagnol ${ }^{1}$

Gabriele Rissotto Menegazzo Luísa Helena do Nascimento Tôrres Jessye Melgarejo do Amaral Giordani ${ }^{1}$

RESUMO: Objetivo:Este estudo trata-se de um relato de experiência acerca de um programa da prefeitura da cidade de Santa Maria, sul do Brasil, vinculado à um projeto de extensão denominado Sorria Santa Maria que teve seu início em 2017 e permanece em andamento. Nosso objetivo com isso foi, além de contribui com os usuários que têm dificuldade em acessar os atendimentos odontológicos na atenção básica, promover e demonstrar as práticas em saúde coletiva, construídas em diferentes processos de trabalho e estreitamente ligadas a estruturas da sociedade.Relato de experiência:O programa destina-se aos usuários do Sistema Único de Saúde do município que não conseguem acessar as Unidades Básicas de Saúde, devido à baixa cobertura de saúde bucal municipal e às funções profissionais exercidas durante horário comercial pelos usuários. Os agendamentos são realizados segundo uma lista de espera com o contato de pacientes que manifestaram desejo de uma avaliação odontológica. Nas consultas, realiza-se então acolhimento, avaliação e atendimento destes pacientes até a conclusão do plano de tratamento. Conclusão:Importantes impactos foram alcançados até o presente momento, tanto em relação ao expressivo número de atendimentos odontológicos realizados quanto à redução das listas de espera para atendimento odontológico. 1 Programa de Pós-Graduação em Ciências Odontológicas, Universidade Federal de Santa Maria, Santa Maria/RS, Brasil 
Além disto, ressaltam-se que as ações de promoção de saúde ligadas ao projeto resultamem uma maior integração entre ensino-serviço-comunidade no município.

Palavras chave: Assistência odontológica, Atenção primária à saúde, Planos e programas de saúde.

ABSTRACT: Objective: This study is an experience report about an extension project linked to a program of the city of Santa Maria, southern Brazil, called Sorria Santa Maria that started in 2017 and remains in progress. Our aim with this was, in addition to contributing with the users who have difficulty accessing dental attendance in primary care, promoting and demonstrating collective health practices, built on different work processes and closely linked to societal structures. Experience report: The program is intended for users of the municipality's Unified Health System who cannot access the Basic Health Units, due to the low coverage of municipal oral health and the professional functions performed during business hours by users. Arranges are made according to a waiting list with the contact of patients who expressed the desire for a dental evaluation. In consultations, these patients are welcomed, evaluated and treated until the treatment plan is completed. Conclusion: Important impacts have been achieved until now, both in relation to the significant number of dental cares, provided and the reduction of waiting lists for dental attendance. In addition, the health promotion actions linked to the project result in greater integration between teaching-service-community in the municipality.

Keywords: Dental care, Health programs and plans, Primary health care.

RESUMEN: Objetivo: Este estudio es un informe de experiencia sobre un proyecto de extensión vinculado a un programa de la ciudad de Santa María, sur de Brasil, llamado Sorria Santa María, que comenzó en 2017 y sigue en progreso. Nuestro objetivo con esto era, además de contribuir a los usuarios que tienen dificultades para acceder a la atención dental en la atención primaria, promover y demostrar prácticas colectivas de salud, basadas en diferentes procesos de trabajo y estrechamente vinculados a las estructuras sociales. Informe de experiencia: El programa está destinado a usuarios del Sistema de Salud Unificado de la municipalidad que no pueden acceder a las Unidades Básicas de Salud, debido a la baja cobertura de salud bucal municipal y las funciones profesionales realizadas por los usuarios durante el horario comercial. Los horarios se hacen de acuerdo con una lista de espera con el contacto de pacientes que expresaron el deseo de una evaluación dental. En las consultas, estos pacientes son bienvenidos, evaluados y tratados hasta que se completa el plan de tratamiento. Conclusión: Hasta ahora se han logrado impactos importantes, tanto en relación con el número significativo de atención dental brindada como con la reducción de las listas de espera para la atención dental. Además, es digno de mención que las acciones de promoción de la salud vinculadas al proyecto dan como resultado una mayor integración entre la comunidad de servicios de enseñanza en el municipio.

Contraseñas: Atención odontológica, Atención primaria de salud, Planes y programas de salud. 


\section{INTRODUÇÃO}

As condições de saúde bucal são reconhecidas como problemas de saúde pública, devido a sua gravidade, prevalência, impacto individual e comunitário, acarretando ao Sistema Único de Saúde (SUS) brasileiro a responsabilidade de desenvolver métodos efetivos de prevenção e tratamento para as mesmas ${ }^{1}$. Considerando a importância das doenças bucais e craniofaciais na carga global de morbidade e sua associação com doenças sistêmicas, a $60^{\circ}$ Assembleia Mundial da Organização Mundial da Saúde (OMS) recomendouaos estados-membros aumentar os investimentos destinados ao cuidado da saúde bucal dos indivíduos². Também deve-se ressaltar que, no Brasil, a saúde bucal está entre os três motivos mais relevantes da demanda por cuidados de saúde 3 .

A condição de saúde bucal da população brasileira teve uma expressiva melhora nas últimas décadas ${ }^{4}$.A primeira pesquisa nacional de saúde bucal, realizadano ano de 1986, mostrou índices elevados de dentes cariados, perdidos ou obturados(CPO-D), cujos valores (média de 6,65para crianças de 12 anos) estavam entre os maiores do mundo ${ }^{5}$. Passados dez anos, realizou-se outro inquérito de saúde bucal, o qual mostrou uma queda do índice CPO-D geral da população de 3,06para 2,786. Em um terceiro inquérito realizado no ano de 2003, evidenciou-se melhora significativa na saúde periodontal da população, todavia, os percentuais de indivíduos com o periodonto saudável ainda eram muito baixos, sendo de 46,18\% para as crianças, $21,94 \%$ para os adultos e 7,89\% para os idosos. Apesar da expressiva melhora na condição de saúde bucal, os valores encontrados no inquérito epidemiológico de 2003 ainda estavam aquém da meta sugerida pela OMS para o ano de 2000. Apenas o índice médio de CPO-D na idade de 12 anos mostrou-se compatível, sendo inferior a 3,0 e mantendo-se com declínio em outro levantamento realizado em nível nacional no ano de 2010, mas variando, todavia, entre as regiões do país ${ }^{7,8,9}$. Neste último levantamento epidemiológico, o índice de dentes cariados e perdidos diminuiu expressivamente na população adulta, dando espaço ao componente obturado, resultado de maior acesso aos serviços para tratamentos odontológicos restauradores. Porém, as diferenças regionais quanto a presença de cárie dentária e o acesso ao serviço foram mantidas9.

Atitudes sociais mais amplas em relação às desigualdades e até mesmo os incentivos e os arranjos de governança no próprio sistema de saúde, tornam mais atraentes a prática de serviços em áreas mais favorecidas ${ }^{10}$, o que leva à uma grande parcela da população a se tornar desassistida. Considerando a importância das barreiras que os usuários enfrentam ao tentar acessar aos serviços de saúde, incluindo o serviço odontológico,essa assistência tem se mostrado, segundo a Pesquisa Nacional de Saúde (PNS) divulgada pelo Instituto Brasileiro de Geografia e Estatística (IBGE), de baixo alcance, sendo que no ano de 2013, 55,6\% dos brasileiros não haviam utilizado o serviço odontológico nos 12 meses anteriores a pesquisa ${ }^{11}$.

Sendo assim,e considerando as metas do Plano Municipal de Saúde Bucal de Santa Maria-RS, como, aumentar a cobertura de saúde bucal e a proporção de pessoas participantes dos procedimentos coletivos de escovação dental supervisionada, foi criadopela Secretaria Municipal de Saúde de 
Santa Maria em abril de 2017, o programa "Sorria Santa Maria" vinculado posteriormente no ano de 2019, à um projeto de extensão da Universidade Federal de Santa Maria. Esteprograma visa contribuir com os usuários que têm dificuldade em acessar os atendimentos odontológicos na atenção básica devido à sua área de cobertura ou ao seu horário de atendimento. E, como as práticas em saúde coletiva são práticas sociais, construídas em diferentes processos de trabalho e estreitamente ligadas a estruturas da sociedade, a vinculação da instituição de ensino nesse serviço se mostra proveitosa para ambas as partes.

Sendo assim, o objetivo do presente trabalho é apresentar um relato de experiência sobre o programa Sorria Santa Maria, promovendo e demonstrando práticas de serviço e gestão em saúde bucal coletiva.

\section{DESENVOLVIMENTO}

O município de Santa Maria localiza-se na região central do estado do Rio Grande do Sul, região sul do Brasil, e possui cerca de 280.505 habitantes, segundo estimativas realizadas pelo Instituto Brasileiro de Geografia e Estatística ${ }^{3}$. O município responde a 4a Coordenadoria Regional de Saúde do Rio Grande do Sul (4 ${ }^{\text {a }}$ CRS/RS), a qual é dividida nas regiões de saúde Verdes Campos e Entre Rios, com Índices de Desenvolvimento Humano considerados altos, baixas taxas de analfabetismo e cobertura da Atenção Primária em Saúde (APS) de 57,9\% e85,9\%, respectivamente ${ }^{12}$.

A Atenção Primária em Saúde no município é composta por 28 Unidades Básicas de Saúde (UBS), distribuídas em Tradicionais (UBST) e Estratégia deSaúde da Família (UBSF), das quais 19 possuem equipe de Saúde Bucal (ESB). O município possui ainda uma Unidade Móvel de Saúde com equipamento odontológico, que realiza atendimentos nas localidades do interior. Na Média Complexidade são três serviços: duas unidades de pronto atendimento e o Centro de Especialidades Odontológicas (CEO), que oferece serviços de periodontia, endodontia, cirurgia bucomaxilofacial, atendimento a pacientes com necessidades especiais e odontopediatria.Na Alta Complexidade são referências em saúde bucal no SUS o Hospital Universitário de Santa Maria (HUSM) e o Hospital Casa de Saúde.

A cobertura populacional estimada pelas equipes básicas de Saúde Bucal no município no ano de criação do projeto era de26,47\%(Sistema de Cadastro de Estabelecimentos de Saúde/ Departamento de Atenção Básica) ${ }^{13}$.

Estudos realizados no município de Santa Maria,na faixa etária de 1 a 5 anos, mostram que condições clínicas e socioeconômicas são fatores determinantes no uso dos serviços odontológicos. A procura por atendimento aumenta com a idade, devido as necessidades de tratamento já estabelecidas, sendo menos comum para prevenção ${ }^{14}$.

Sendo assim, considerando a baixa cobertura em saúde bucal no município, a existência de lista de espera para atendimento odontológico proveniente das unidades sem equipes de saúde bucal, 
bem como o horário de funcionamento das unidades de saúde, a Coordenação da Política de Saúde Bucal de Santa Maria propôs o programa“Sorria Santa Maria"15.

O programa tem como objetivo auxiliar na redução dos índices de doenças e agravos em saúde bucal dos usuários do SUS, facilitando o acesso aos atendimentos odontológicos e realizando orientações de higiene bucal, prevenção e tratamento odontológico para trabalhadores, gestantes e crianças, a fim de proporcionar a essa população uma condição de saúde bucal adequada e melhor qualidade de vida. Também visa oportunizar vivência e integração entre o ensino e o serviço, no intuito de corroborar com a qualidade de aprendizagem dos envolvidos através da educação permanente e estar vinculado à um projeto de extensão da Universidade Federal de Santa Maria (UFSM), onde alunos de graduação e pós-graduação em saúde coletiva participam ativamente das atividades.

O Projeto desenvolve-se nas dependências do Centro de Especialidades Odontológicas, mensalmente aos sábados. A participação dos usuários no projeto se dámediante agendamento, o qual é realizado pelo contato de pacientes que manifestaram desejo de uma avaliação odontológica e estão arrolados em uma lista de espera para atendimento odontológico, bem como pelo acolhimento a demanda espontânea. O público alvo são os usuários que não possuem equipe de saúde bucal de referência nas unidades de saúde onde são cadastrados, ou não conseguem acessar a unidade em horário comercial em função das suas atividades laborais, além de gestantes que não estavam realizando pré-natal odontológico pelos mesmos motivos, e crianças desassistidas.

Na primeira consulta, o usuário é acolhido, avaliado e tem seu plano de tratamento elaborado. A partir de então, o retorno é garantido até a conclusão do tratamento, incluindo o encaminhamento para especialidades odontológicas ofertadas pelo Centro de Especialidades Odontológicas municipal quando necessário, garantindo a integralidade do cuidado. Os procedimentos, bem como a organização e planejamento do projeto, são realizados pelos cirurgiões-dentistas e auxiliares de saúde bucal da Rede de Atenção à Saúde do município, bem como por residentes da residência multiprofissional do Hospital Universitário de Santa Maria e pós-graduandos do Programa de PósGraduação em Ciências Odontológicas com ênfase em Saúde Coletiva da UFSM.

Os usuários atendidos no programa possuem acesso a procedimentos odontológicos ofertados pela atenção básica como raspagens periodontais, restaurações, exodontias, entre outros. Também é realizado o pré-natal odontológico, onde cirurgião-dentista acompanha o estado de saúde bucal das gestantes a fim de promover saúde, evitar o agravamento de problemas já instalados, prevenir outras doenças e orientar quanto a importância da manutenção da saúde bucal nesse período assim como a transmissão de hábitos saudáveis para o bebê.

O primeiro ano de atividade do programa "Sorria Santa Maria" foi 2017 e contou com 14 edições, nas quais foram realizados 1.762 procedimentos, sendo 213 destes em gestantes. Em 2018, foram realizadas 10 edições do programa com 1.649 procedimentos, sendo 258 realizados 
em crianças e 86 em gestantes. A maior parte dos procedimentos realizadosforam de caráter preventivo, englobando orientações de higiene oral, limpeza profilática e aplicação tópica de flúor. Atualmente, o programa segue sendo executado mensalmente.

Além dos atendimentos odontológicos, são realizadas ações que visam à promoção de saúde bucal nas suas atividades, como orientações sobre alimentação saudável, higiene oral adequada e realização de escovação supervisionada. Elaboram e conduzem essas ações acadêmicos e professores da UFSM e estagiários do município, proporcionando a aproximação entre as instituições e o intercâmbio de saberes e práticas. Também são realizadas ações integradas junto a outros núcleos profissionais com o intuito de promover saúde para a população, das quais destacamse salas de espera com temáticas abordando saúde da mulher, doenças crônicas, ações da política de saúde HIV/AIDS, prevenção do câncer de boca, práticas integrativas e complementares com a aplicação de reiki e acupuntura, entre outras.

\section{CONSIDERAÇÕES FINAIS}

Com a baixa cobertura de serviços de saúde bucal e o aumento da demanda por cuidados bucais, o programa "Sorria Santa Maria" vem sendo uma alternativa que tem contribuído para redução dos índices de agravos em saúde bucal do município, aumentando o acesso aos atendimentos odontológicos, pautado na integralidade do cuidado e promovendo vínculo entre ensino, pesquisa, extensão, serviço e gestão.Somando a isso, o Sorria Santa Maria oportunizou que gestantes sem referência de saúde bucal no seu território realizassem o pré-natal odontológico, recebendo cuidadosde saúde bucal e geral, com a continuidade após o parto.

Embora o programa seja efetivo, e tenha contribuído de forma a minimizar os problemas de saúde bucal locais, é importante que maiores investimentos sejam realizados no setor, a fim de aumentar a cobertura populacional de serviços de saúde bucal nomunicípio e o número de profissionais de saúde bucal, facilitando dessa forma o acesso da população.

\section{REFERÊNCIAS BIBLIOGRÁFICAS}

1. Antunes JLF, Toporcov TN, Bastos JL, Frazão P, Narvai PC, Peres MA. Oral health in the agenda of priorities in public health. Rev Saúde Públ. 2016;50(0).

2. Organização Mundial da Saúde. Oral health surveys: basic methods. In. Geneva, 1997:93.

3. Instituto Brasileiro de Geografia e Estatística. Síntese de Indicadores Sociais. Uma análise das condições de vida da população brasileira. 2010.

4. Pucca GA, Gabriel M, de Araujo ME, de Almeida FCS. Ten Years of a National Oral Health Policy in Brazil: Innovation, Boldness, and Numerous Challenges. J Dent Res.outubro de 2015;94(10):1333-7. 
5. Oliveira AGRC. Perfil epidemiológico de saúde bucal no Brasil 1986-1996. Brasil. 2001. [Acessado 2019 Ago 12]. Disponível em: http://paginas.terra.com.br/saude/angelonline/artigos/ art_epid/epi_bra.pdf

6. Oliveira AGRC. Levantamento epidemiológico em saúde - bucal $1^{\mathrm{a}}$ etapa - Cárie Dental, 1996. Relatório Paralelo. 1998. [acessado 2019Ago 2]: [cerca de 20 p.]. Disponível em: http://www. angelonline.cjb.net.htm

7. Brasil. Ministério da Saúde. Projeto SB Brasil 2003 Condições de Saúde Bucal da População Brasileira 2002-2003. Resultados principais. 2003. [acessado 2019Ago 01] Disponível em: http:// www.cfo.org.br/download/pdf/relatorio_sb_brasil_2003.pdf

8. Freire $\mathrm{M}$ do CM, Reis SCGB, Figueiredo N, Peres KG, Moreira R da S, Antunes JLF. Determinantes individuais e contextuais da cárie em crianças brasileiras de 12 anos em 2010. Rev Saúde Públ. 2013;47(suppl 3):40-9.

9. Brasil. Ministério da Saúde. Secretaria de Atenção à Saúde. Secretaria de Vigilância em Saúde. SB Brasil 2010: Pesquisa Nacional de Saúde Bucal: resultados principais. Brasília: Ministério da Saúde, 2012.

10. Harris RV, Pennington A, Whitehead M. Preventive dental visiting: a critical interpretive synthesis of theory explaining how inequalities arise. Community Dent Oral Epidemiol. 2017;45(2):120-34.

11. Instituto Brasileiro de Geografia e Estatística. Síntese de Indicadores Sociais. Uma análise das condições de vida da população brasileira. 2015.

12. Instituto Brasileiro de Geografia e Estatística. Síntese de Indicadores Sociais. Uma análise das condições de vida da população brasileira. 2012.

13. Carrer, FCA, SUS e Saúde Bucal no Brasil: por um futuro com motivos para sorrir. São Paulo : Faculdade de Odontologia da USP, 2019. 167p

14. Ardenghi TM. Age of first dental visit and predictors for oral healthcare utilization in preschool children. Oral Health Prev Dent. 2012;10(1):17-27.

15. Mazzorani BM, Duarte L de M, Socal ES, Martins V. Núcleo de Coordenação do Conselho Municipal de Saúde. Plano Municipal de Saúde 2018-2021. Santa Maria. 2018:103. po

Artigo apresentado em outubro de 2019 Artigo aprovado em janeiro de 2020 Artigo publicado em maio de 2020 